\title{
Difluprednate for the prevention of ocular inflammation postsurgery: an update
}

This article was published in the following Dove Press journal:

Clinical Ophthalmology

16 June 2011

Number of times this article has been viewed

\section{Eric D Donnenfeld}

Ophthalmic Consultants of Long Island, Rockville Centre, NY, USA

Correspondence: Eric D Donnenfeld Ophthalmic Consultants of Long Island, 2000 North Village Avenue, Suite 402, Rockville Centre, NY I I570, USA

$\mathrm{Tel}+\mathrm{I} 5167662519$

Fax+I 51676637|4

Email eddoph@aol.com
Purpose: To review the most recent published data regarding the novel potent steroid, difluprednate ophthalmic emulsion, $0.05 \%$.

Methods: A comprehensive search of recent published literature including difluprednate was performed. Clinical studies relevant to the characteristics and clinical efficacy of difluprednate in controlling postoperative inflammation were included, and a synopsis of each study was developed.

Results: Several recent publications were identified in which difluprednate was shown to be efficacious in the treatment of postoperative inflammation in different clinical settings, including a novel perioperative regimen. Additional literature retrieved from this search included data on the relative potency of difluprednate, potential utility in the posterior segment, as well as the advantages of the emulsion formulation.

Conclusion: Difluprednate has been studied extensively and shown in recent literature to be a safe and effective topical anti-inflammatory drug. The proven strength and unique formulation of difluprednate, along with its potent efficacy in treating and preventing inflammation, provides clinicians with a beneficial treatment option.

Keywords: corticosteroids, anti-inflammatory, dose uniformity, relative potency

\section{Introduction}

Controlling and preventing inflammation is the most important concern of the ophthalmologist in achieving optimal results following surgery. The physician can proactively help to reduce the risk of inflammation that can occur after the operation takes place. Even though some ocular surgeries, such as phacoemulsification, do not generally result in significant inflammation, there are still a portion of patients that will experience some form of postoperative inflammation, which can potentially lead to sight-threatening issues such as cystoid macular edema. ${ }^{1}$ As such, the majority of physicians employ a prophylactic regimen of anti-inflammatory medications in the perioperative period. Because of their broad anti-inflammatory activity, corticosteroids are typically the cornerstone of these treatment regimens.

Almost 3 years ago, a potent new steroid - difluprednate ophthalmic emulsion, $0.05 \%$ (Durezol ${ }^{\circledR}$, Alcon Laboratories, Inc, Fort Worth, TX) - was approved by the US Food and Drug Administration for the treatment of inflammation and pain associated with ocular surgery. ${ }^{2}$ Since then, many physicians have had extensive clinical experience with difluprednate and have incorporated it into their standard anti-inflammatory treatment regimen. Because of the increased awareness and subsequent use of this steroid, many studies exploring the characteristics and efficacy of difluprednate have 
recently been published. This review will provide a summary of recent publications covering dose uniformity, relative potency, and clinical utility of this new steroid in different clinical settings.

\section{Difluprednate dose uniformity}

Due to the lipophilic nature of steroid molecules, and therefore their inability to dissolve in a solution, the majority of steroid preparations are formulated as suspensions. Ophthalmic suspensions tend to settle over time, and as a result, must be shaken by the patient prior to use to ensure a homogenous distribution of active drug within the aqueous phase of the suspension. If a bottle of a suspension formulation is not shaken, the drops may have subtherapeutic levels of corticosteroid, even in the first days postoperatively when anti-inflammatories are typically needed most. Unfortunately, as most clinicians know, patients are not very compliant when it comes to shaking their medication. This was borne out of a study of steroid preparations, in which only $37 \%$ of patients followed the prescribed shaking instructions. ${ }^{3}$ In addition, even when patients appropriately shake their medication, particles within the suspensions still have a tendency to agglomerate or cake, especially as the particle size of the drug increases. The potential lack of homogeneity with suspensions can lead to dosage inconsistencies. Difluprednate ophthalmic emulsion was formulated as a stable oil-in-water emulsion to achieve optimum dosage consistency. Stringer and Bryant conducted a study to determine whether the position and shaking of a bottle affected the amount of active ingredient delivered. ${ }^{4}$ Three topical steroids - difluprednate ophthalmic emulsion, generic prednisolone acetate suspension 1\% (Falcon Pharmaceuticals, Ltd, Fort Worth, TX), and branded prednisolone acetate suspension 1\% (Pred Forte ${ }^{\circledR}$, Allergan, Inc, Irvine, CA) - were stored upright and either shaken or not shaken, or were stored upside down and not shaken. Two drops were dosed four times a day to simulate patient use, and were then measured for the amount of active drug delivered in each drop. The dose uniformity of difluprednate was excellent regardless of bottle storage position or shaking before use. All doses were close to $100 \%$ of the stated label claim.

Generic prednisolone acetate suspension exhibited very poor dose uniformity, and after inverted storage and no shaking trended from very high concentrations (more than $700 \%$ of label claim) on day 1 to very low concentrations after 1 week, most of which were subtherapeutic levels. Even in ideal conditions - stored upright and shaken - it demonstrated significant variability in dose concentration. The branded version of prednisolone acetate also exhibited similar trends, although not as extreme. Table 1 shows the percentage of drops that were within $15 \%$ of declared concentration for all conditions tested. All doses of difluprednate remained within this range $100 \%$ of the time, no matter the orientation or handling of the bottle. In contrast, generic prednisolone acetate concentrations were within $15 \%$ of declared concentration for only $4 \%-13 \%$ of the time points, depending on the condition tested.

Difluprednate ophthalmic emulsion demonstrated excellent and consistent dose uniformity compared with the suspensions, suggesting that the clinical use of difluprednate may produce more predictable efficacy and safety.

\section{Difluprednate potency}

Glucocorticoid receptor-binding activity (GCRBA) is an important measure widely accepted as an index of pharmacologic effect. Tajika et al conducted a study to determine the GCRBA of difluprednate ophthalmic emulsion and its active metabolite, $6 \alpha$,9-difluoroprednisolone 17-butyrate (DFB), compared with prednisolone, betamethasone, fluorometholone, and dexamethasone. ${ }^{5}$ A glucocorticoid receptor-binding test was performed to evaluate the $\mathrm{K}_{\mathrm{i}}$ value of each steroid, which is an inhibition constant that indicates the affinity for a receptor. A lower $\mathrm{K}_{\mathrm{i}}$ value indicates a stronger affinity for the glucocorticoid receptor. The results of this study most notably showed that DFB, the active metabolite of difluprednate ophthalmic emulsion, had the lowest $\mathrm{K}_{\mathrm{i}}$ value $\left(6.11 \times 10^{-11} \mathrm{~mol} / \mathrm{L}\right)$ and was significantly more active than prednisolone, which had a $\mathrm{K}_{\mathrm{i}}$ value of $3.4 \times 10^{-9} \mathrm{~mol} / \mathrm{L}$. This demonstrates that the glucocorticoid binding affinity for the active metabolite of difluprednate was 56 times stronger than prednisolone.

The greater binding affinity may be attributed to the unique molecular structure of difluprednate. Difluprednate is a derivative of prednisolone but differs substantially due to structural modifications. The first is the addition of fluorine

Table I Percentage of data points within 15\% of declared concentration $^{4}$

\begin{tabular}{llll}
\hline Usage condition & Pred Forte $^{\circledR}$ & Durezol $^{\circledR}$ & $\begin{array}{l}\text { Generic } \\
\text { prednisolone }\end{array}$ \\
\hline Upright, not shaken & $54 \%$ & $100 \%$ & $13 \%$ \\
Upright, shaken & $40 \%$ & $100 \%$ & $6 \%$ \\
Inverted, not shaken & $0 \%$ & $100 \%$ & $4 \%$ \\
\hline
\end{tabular}

Copyright @ 2010 , Stringer and Bryant. ${ }^{4}$ Reproduced with permission from Dove Medical Press. 
atoms at the C-6 and C-9 positions. It is well-known that fluorination of corticosteroids greatly increases specificity for the glucocorticoid receptor ${ }^{6}$ and many of the morepowerful glucocorticoids are fluorinated at either the C-6 or C-9 position (eg, betamethasone, clobetasol), or at both (eg, fluticasone). Difluprednate is the only difluorinated topical ophthalmic glucocorticoid available in the US. The second modification is the addition of a butyrate ester at the $\mathrm{C}-17$ position. This has also been shown to further increase the potency of difluprednate. ${ }^{7}$ Thus, the first two modifications - the addition of the two fluorines and of the C-17 butyrate - directly increase the affinity of difluprednate for the glucocorticoid receptor. While receptor binding is important, it is not the only determinant of therapeutic effectiveness. In the case of a topical ophthalmic steroid, the drug must also have access to the receptor at the site of inflammation. The third modification of difluprednate increases its ability to reach the relevant ocular glucocorticoid receptors. Previous work has shown that the corneal penetration of glucocorticoids is enhanced by the addition of an acetate group to the molecule. ${ }^{8}$ In difluprednate, the addition of the acetate ester at position $\mathrm{C}-21$ enhances tissue penetration, enabling more active drug to reach the uvea. In fact, glucocorticoids with 17,21-double esters, such as difluprednate, generally penetrate tissue better than monoester derivatives. ${ }^{9}$

\section{Safety}

All ophthalmic corticosteroids, both topical and systemic, have the potential to provoke a rise in intraocular pressure (IOP), especially in patients with certain risk factors, such as myopia, diabetes, and family history of glaucoma. Genetic factors likely also exist that confer a risk of IOP elevation secondary to corticosteroid exposure. Difluprednate is no different in this regard; that is, it can also be associated with elevated IOP. Thus, standard of care practices must be employed, with frequent measurement of eye pressure for anyone using this medication.

As a general rule, the more powerful the steroid, the more prevalent the adverse events. In several clinical trials where Durezol was dosed either QID or BID for a median time of 27.1 days, a $3 \%-6 \%$ rate of clinically significant IOP elevation was evident, defined as a rise in IOP of more than $21 \mathrm{mmHg}$ and at least $10 \mathrm{mmHg}$ from baseline at the same visit. While this rate is in line with other moderate-tostrong steroids, there have been anecdotal and unpublished accounts of pressure increases that are of greater intensities than what is typically seen with this class. However, its enhanced potency, which permits less frequent dosing than prednisolone, and its emulsion formulation, which does not require shaking the bottle to resuspend the drug, make difluprednate a major advancement in patient compliance and inflammatory control. Difluprednate is highly likely to become the preferred product for most eye care specialists who manage all types of intraocular inflammation, despite the universal caution of IOP increase that accompanies all steroid use.

\section{Difluprednate in postoperative inflammation}

Recently, my colleagues and I conducted a study testing a novel steroid pulse-dosing regimen. This study was designed based upon the use of steroids in other medical disciplines outside of ophthalmology. In a large systematic review that analyzed numerous randomized controlled clinical trials involving major surgical procedures, the administration of a steroid prior to surgery reduced local edema and pain in many of the procedures. ${ }^{10}$ It was found that the timing of steroid administration was critically important - steroids dosed less than 1-2 hours prior to surgery seemed to have less effect, potentially because the protein-mediated onset of action for steroids takes about this long. In addition, the large National Acute Spinal Cord Injury trial demonstrated that the administration of high-dose steroids immediately after surgery helps to preserve and protect neural tissue. ${ }^{11}$ Based on these two concepts, my colleagues and I tested a regimen in which two steroids (difluprednate ophthalmic emulsion and prednisolone acetate suspension) were dosed at least 10 times on the day of cataract surgery, and then continued for 2 weeks postoperatively. ${ }^{12}$ Our study was a prospective multicenter, double-masked, randomized, contralateral-eye trial conducted in 63 patients (126 eyes). We found that the administration of difluprednate in this pulse-dosing fashion provided better vision and less corneal edema (measured via pachymetry) on day 1 when compared with prednisolone. On the first day after surgery, 19 patients in the difluprednate arm and six patients in the prednisolone arm had a best corrected visual acuity (BCVA) of 20/20. Table 2 shows the uncorrected visual acuity (UCVA) and BCVA at day 1 for the two regimens.

The mean central corneal thickness at day 1 in the difluprednate group increased $28 \mu \mathrm{m}$ (from 562 to $590 \mu \mathrm{m}$ ). This increase was about half of that observed in the prednisolone group, which was $57 \mu \mathrm{m}$ (from 562 to $619 \mu \mathrm{m}$ ). Additionally, central corneal thickness at day 1 in the difluprednate 
Table 2 UCVA and BCVA at day I in each treatment arm

\begin{tabular}{|c|c|c|c|c|c|c|}
\hline \multirow[t]{3}{*}{ Snellen visual acuity } & \multirow{2}{*}{\multicolumn{3}{|c|}{$\begin{array}{l}\text { UCVA at day I } \\
\text { Number of subjects (percentage of subjects) }\end{array}$}} & \multirow{2}{*}{\multicolumn{3}{|c|}{$\begin{array}{l}\text { BCVA at day I } \\
\text { Number of subjects (percentage of subjects) }\end{array}$}} \\
\hline & & & & & & \\
\hline & Difluprednate & Prednisolone & $P$-value & Difluprednate & Prednisolone & $P$-value \\
\hline $20 / 20$ or better & $8(16 \%)$ & $2(4 \%)$ & NS & $19(38 \%)$ & $6(12 \%)$ & $P<0.005$ \\
\hline $20 / 30$ or better & $28(56 \%)$ & $20(40 \%)$ & NS & $43(86 \%)$ & $28(56 \%)$ & $P<0.005$ \\
\hline $20 / 40$ or better & $36(72 \%)$ & $32(64 \%)$ & NS & 48 (96\%) & $4 \mathrm{I}(82 \%)$ & $P<0.05$ \\
\hline $20 / 70$ or better & 47 (94\%) & 44 (88\%) & NS & $50(100 \%)$ & 47 (94\%) & NS \\
\hline $20 / 100$ or better & $50(100 \%)$ & 48 (96\%) & NS & $50(100 \%)$ & 48 (96\%) & NS \\
\hline
\end{tabular}

Abbreviations: BCVA, best corrected visual acuity; NS, not significant; UCVA, uncorrected visual acuity.

group averaged $32.59 \mu \mathrm{m}$ less than that of the prednisolone group $(P=0.026)$. At day 1 , corneal swelling in the difluprednate group was $31.79 \mu \mathrm{m}$ less than the prednisolone group ( $P=0.033)$ compared with baseline.

One of the important secondary endpoints assessed was endothelial cell counts. In the difluprednate arm, there was significantly less endothelial cell loss at day 30 (Figure 1), with a difference of 180 cells between the two groups. This was interesting, since it shows that a pulse-dosing regimen may help to preserve endothelial cells, and that a more potent steroid may have an added effect.

In addition, OCT measurements revealed thinner retinas at days 15 and 30 in the difluprednate group (Figure 2). By day 15 , the mean retinal thickness in the eyes treated with difluprednate was $7.74 \mu \mathrm{m}$ less than the prednisolone treated eyes $(P=0.011)$. Similarly, by day 30 , the mean retinal thickness of the difluprednate group was $5 \mu \mathrm{m}$ less than the prednisolone group. This study not only demonstrates the utility of this pulse-dosing regimen, but also demonstrates the clinical potential of this new topical steroid. Furthermore, a recent anterior uveitis study conducted by Foster et al showed that difluprednate dosed four times a day exhibited a potent anti-inflammatory effect when compared with branded prednisolone acetate (Pred Forte ${ }^{\circledR}$ ) dosed eight times a day. ${ }^{13}$ We now have a steroid that achieves similar clinical results at half the dose or less of our old standard treatment.

\section{Difluprednate in the posterior segment}

In a recently published preclinical study by Tajika et al, a single instillation of radiolabeled difluprednate resulted in detectable posterior segment levels (anterior retina/ choroid = $273 \mathrm{ngeq} / \mathrm{g}$; posterior retina $/$ choroid $=59 \mathrm{ngeq} / \mathrm{g}$ ), suggesting that topical administration may have possible effectiveness in the posterior segment. ${ }^{14}$ While no large studies have specifically explored the use of difluprednate in retinal disease, two small case control studies published by Nakano et al demonstrate potential utility. In the first, Durezol was compared with a sub-Tenons injection of triamciniolone and was found to have similar effects at reducing retinal thickness in patients with refractory diabetic macular edema (DME). ${ }^{15}$ In the second, Durezol was compared with betamethasone

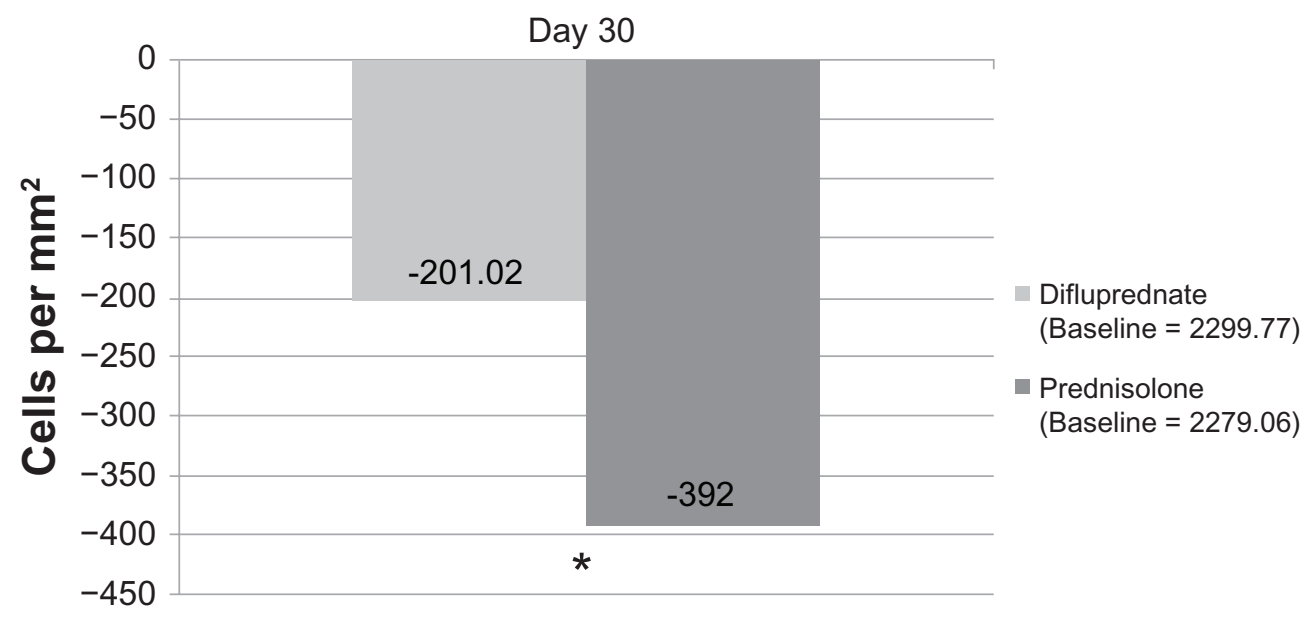

Figure I The change from baseline endothelial cell counts of eyes treated with difluprednate or prednisolone. Note: $*$ Denotes $P=0.0036, t$-test between mean baseline differences of fellow eyes. 


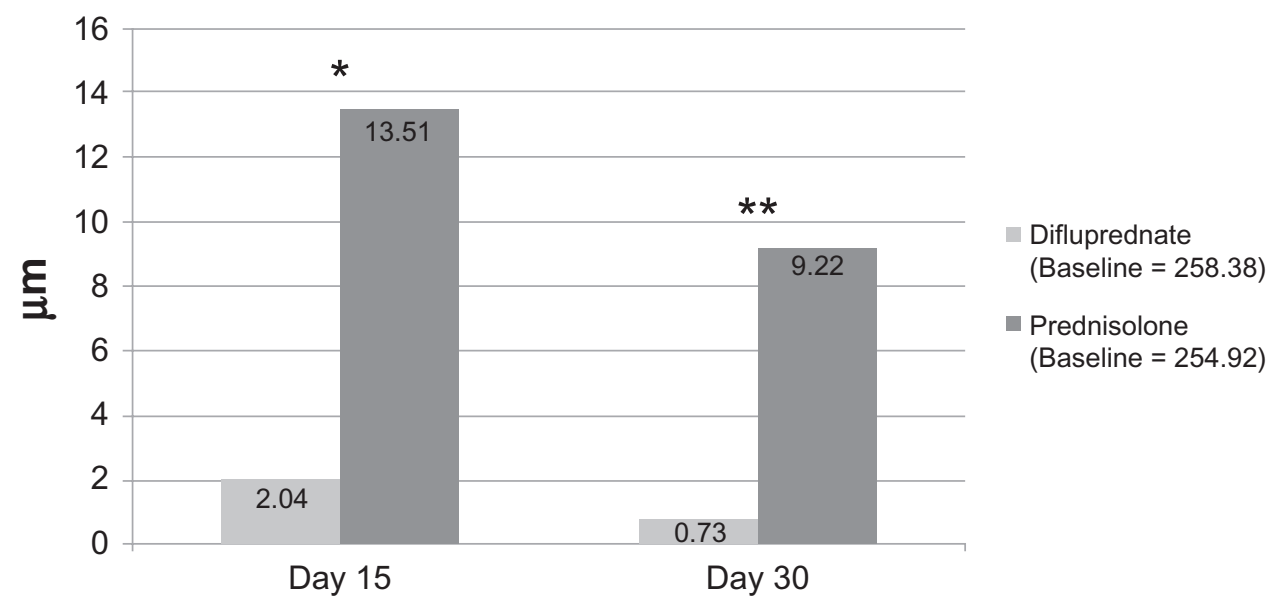

Figure 2 The change from baseline in retinal thickness of eyes treated with difluprednate or prednisolone.

Notes: *Denotes $P<0.0001$ and $* *$ denotes $P=0.0041$, $t$-test between mean baseline differences in fellow eyes.

in treating diffuse DME prior to vitrectomy, and Durezol was found to reduce retinal thickness and improve VA more effectively than betamethasone after 1 month of treatment. ${ }^{16}$ While these studies must be interpreted cautiously, due to their size and design, they provide interesting hypotheses. Nonetheless, given Durezol's high potency and strong affinity for the glucocorticoid receptor, there may be a place for this topical steroid in treating posterior segment disease with an inflammatory component.

\section{Conclusion}

While several steroids have been introduced over the last few decades, difluprednate is the first to be more potent than prednisolone acetate, which has been considered the "gold standard" and indeed has enjoyed a status as the "go-to" steroid for many inflammatory conditions.

Although cataract surgery complications are generally rare, approximately $5 \%$ of patients will experience problems after surgery, ranging from mild and transient conditions to more sight-threatening issues that require further treatment. ${ }^{1}$ During the past decade, advances in cataract surgery techniques, equipment, and pharmacologic strategies have helped to keep the rate of complications low. Nonetheless, complications still occur, and in an effort to prevent these, most physicians will prophylactically treat patients with a standard perioperative regimen to prevent infections and reduce postoperative inflammation and pain.

With the proven efficacy of difluprednate, as is evident from these reviewed publications, we now have a new standard for potency in a topical corticosteroid, with excellent anti-inflammatory properties and an ideal formulation for our patients.

\section{Disclosure}

The author reports no conflicts of interest in this work.

\section{References}

1. El-Harazi SM, Feldman RM. Control of intra-ocular inflammation associated with cataract surgery. Curr Opin Ophthalmol. 2001;12(1): 4-8.

2. Durezol ${ }^{\circledR}$ (difluprednate ophthalmic emulsion, $0.05 \%$ ) Package Insert Alcon Laboratories, Inc, Fort Worth, TX.

3. Apt I, Hendrick A, Silverman L. Patient compliance with use of topical ophthalmic corticosteroid suspensions. Am J Ophthalmol. 1979; $87(2): 210-214$.

4. Stringer W, Bryant R. Dose uniformity of topical corticosteroid preparations: difluprednate ophthalmic emulsion $0.05 \%$ versus branded and generic prednisolone acetate ophthalmic suspension 1\%. Clin Ophthalmol. 2010;5(4):1119-1124.

5. Tajika T, Waki M, Tsuzuki M, Kida T, Sakaki H. Pharmacokinetic features of difluprednate ophthalmic emulsion in rabbits as determined by glucocorticoid receptor-binding bioassay. J Ocul Pharmacol Ther. 2011;27(1):29-34

6. Bush IE, Mahesh VB. Metabolism of 11-oxygenated steroids. 3. Some 1-dehydro and 9 alpha-fluoro steroids. Biochem J. 1964;93(2): 236-255.

7. Wang RIH. Sytkowski A, Shetty KR. Relating corticosteroid structure and function. Drug Ther. 1975;143-148.

8. Bartlett JD, Bennett ES, Fiscella RG, Jaanus SD, Zimmerman TJ. Ophthalmic Drug Facts. 19 ed. St Louis, MO:Wolters Kluwer; 2008.

9. Hammer S, Spika I, Sippl W, et al. Glucocorticoid receptor interactions with glucocorticoids: evaluation by molecular modeling and sdfsdf functional analysis of glucocorticoid receptor mutants. Steroids. 2003; 68(4):329-339.

10. Holte K, Kehlet H. Perioperative single-dose glucocorticoid administration: pathophysiologic effects and clinical implications. J Am Coll Surg. 2002;195(5):694-712.

11. Bracken MB, Shepard MJ, Holford TR, et al. Methylprednisolone or tirilazadmesylate administration after acute spinal cord injury: 1-year follow up. Results of the third National Acute Spinal Cord Injury randomized controlled trial. J Neurosurg. 1998;89(5):699-706.

12. Donnenfeld ED, Holland EJ, Solomon KD, et al. A multicenter randomized controlled fellow eye trial of pulse dosed difluprednate $0.05 \%$ versus prednisolone acetate $1 \%$ in cataract surgery. Am J Ophthalmol. In press 2011. 
13. Foster CS, Davanzo R, Flynn TE, McLeod K, Vogel R, Crockett RS. Durezol $^{\circledR}$ (difluprednate ophthalmic emulsion $0.05 \%$ ) compared with Pred Forte ${ }^{\circledR} 1 \%$ ophthalmic suspension in the treatment of endogenous anterior uveitis. J Ocul Pharmacol Ther. 2010;26(5):475-483.

14. Tajika T, Isowaki A, Sakaki H. Ocular distribution of difluprednate ophthalmic emulsion $0.05 \%$ in rabbits. J Ocul Pharmacol Ther. 2011; 27(1):43-49.
15. Nakano S, Yamamoto T, Kirii E, Abe S, Yamashita H. Steroid eye drop treatment (difluprednate ophthalmic emulsion) is effective in reducing refractory diabetic macular edema. Graefes Arch Clin Exp Ophthalmol. 2010;248(6):805-810.

16. Nakano Goto S, Yamamoto T, Kirii E, Abe S, Yamashita H. Treatment of diffuse diabetic macular oedema using steroid eye drops. Acta Ophthalmol. [Epub ahead of press].

\section{Publish your work in this journal}

Clinical Ophthalmology is an international, peer-reviewed journal covering all subspecialties within ophthalmology. Key topics include: Optometry; Visual science; Pharmacology and drug therapy in eye diseases; Basic Sciences; Primary and Secondary eye care; Patient Safety and Quality of Care Improvements. This journal is indexed on

\section{Dovepress}

PubMed Central and CAS, and is the official journal of The Society of Clinical Ophthalmology (SCO). The manuscript management system is completely online and includes a very quick and fair peer-review system, which is all easy to use. Visit http://www.dovepress.com/ testimonials.php to read real quotes from published authors. 